\title{
Analisi sistematica degli insediamenti cicladici attraverso un gis multi-layers
}

\author{
Serena Torello Di Nino
}

\section{Il Bronzo Antico come presupposto}

La Civiltà Cicladica nasce tra la fine del Neolitico Recente e l'inizio del Bronzo Antico, periodo che vede la massima fioritura fino al 2000 a.C.

Il passaggio tra Neolitico Recente e Bronzo Antico è visto come una transizione abbastanza netta, che presuppone una decisa soluzione di continuità sia culturale che insediamentale. Sicuramente la rottura fra queste due fasi è dovuta all'introduzione della metallurgia e al processo di urbanizzazione, che sono due delle caratteristiche principali della prima età del Bronzo; la quale, nell'area egea, rappresenta un importantissimo momento di formazione culturale che influenzerà ben presto tutto il bacino mediterraneo orientale. Le aree egee, anatoliche e del futuro continente greco sviluppano in questo momento forme culturali autonome ma fortemente a contatto, mostrando allo stesso tempo una forte differenziazione per aree geografiche, ma anche lineamenti comuni frutto di un sincretismo culturale dovuto a contatti, commerci, viaggi di merci, uomini e idee.

È necessario fornire una cronologia esauriente $e^{1}$ della prima età del bronzo antico che risulta articolato in tre sottofasi:

- BAI 3000-2600 a.C.

- BAII 2600-2300 a.C.

- BAIII A 2300-2000 a.C. e BAIII B 2000-1800 a.C.

Possono essere riconosciuti almeno tre fattori che favorirono la fioritura della Civiltà Cicladica nel corso del III millennio a.C.: la posizione geografica delle isole, le loro caratteristiche climatiche e ambientali e la ricchezza delle materie prime.

Le Cicladi, per la loro posizione geografica, costituirono un punto nevralgico per i contatti tra la Grecia continentale e l'Anatolia, cioè tra l'Europa e l'Asia, ma anche tra Creta e il continente greco, in altre parole un punto di comunicazione tra i popoli dell'Egeo. Una forte vocazione commerciale ha favorito lo sviluppo della cultura cicladica attraverso un commercio di cabotaggio da un'isola all'altra.

I limiti imposti dall'ambiente e dalle risorse naturali spinsero gli abitanti, fin dai tempi più remoti, a volgersi verso il mare e a trasformarsi, con il passare del tempo, in esperti navigatori, abili commercianti e intraprendenti protagonisti degli scambi di materie prime, manufatti, conoscenze e idee da un angolo all'altro dell'arcipelago. Grazie alla ricchezza delle risorse naturali, le Cicladi divennero una delle fonti principali di approvvigionamento di materie prime nell'ambito dell'Egeo: l'ossidiana di Melos, lo smeriglio di Naxos, il piombo e l'argento di Sifnos, il rame e lo stagno di Serifos, oltre al cristallino marmo di Paros e Naxos ${ }^{2}$.

La Civiltà Cicladica, considerata nei suoi più caratteristici lineamenti culturali, assume una sua spiccata individualità nel $\mathrm{BAI}^{3}$ e vede la nascita delle tre culture cicladiche: GrottaPelos, Keros-Syros e Phylakopi I ${ }^{4}$ (Tav. 1).

La cultura di Grotta-Pelos sembra essere quella più antica, non sono state trovate attestazioni nel nord delle Cicladi, ad esempio come a Syros e Mikonos, invece scarse sono le attestazioni

\footnotetext{
${ }^{1}$ BARBER 1977, p.21.

2 BARBER, MACGILLIVRAY 1980, pp.141-157.

${ }^{3}$ BROODBANK 2000.

${ }^{4}$ DOUMAS 1977.
} 
a Paros, Antiparos, Melos, Naxos, Siphnos, Amorgos e Keros ${ }^{5}$. Le maggiori caratteristiche di questi siti si riscontrano essenzialmente nel tipo di ceramica, in cui prevale la ciotola con spesso bordo e ansa orizzontale, ma anche nel tipo di tombe. La maggior parte di queste contengono una sola sepoltura, ma sono state attestate anche tombe doppie che sono un'indicazione di tombe multiple. Le tombe sono solitamente del tipo detto 'a cista' e consistono in quattro lastre verticali poste in una sorta di sistemazione con intento di sostruzione e allo stesso tempo monumentalizzazione della fossa, ed una quinta che funge da copertura. Esse di norma non presentano un orientamento definito.

Con la cultura di Keros-Syros possiamo iniziare a parlare dell'età dei metalli nell'Egeo. Solo ora si hanno numerosi oggetti in metallo e sono spesso accompagnati da una serie di novità, a partire dalle nuove forme ceramiche che si rifanno anche a quelle presenti nella Grecia continentale e all'Anatolia. La cultura di Keros-Syros si differenzia dalla precedente oltre che per i manufatti anche per la cronologia, siamo infatti nel BAII, e per l'area di distribuzione, che non risulta affatto complementare alla prima; sono state trovate, infatti, attestazioni a Thera, Naxos e Siphnos (solo nel periodo di transizione tra BAII e BAIII), Amorgos, e Mikonos.

All'interno delle tombe è stato trovato materiale che si differenzia dalla cultura di Grotta-Pelos. Le pissidi sono lenticolari e non cilindriche, i piedi delle giare sono differenti ed inoltre la superficie rosso-marrone della ceramica è lucida.

Sicuramente la novità per eccellenza è il fatto che nel BAII gli insediamenti assumono un aspetto di cittadelle fortificate, come il sito di Kastri o Panormos (cfr. infra), rispettivamente a Syros e Naxos.

La cultura di Phylakopi è, sicuramente, la meglio indagata e la meglio datata. Già nel BAI si parla di una 'Phylakopi Pre-City' (3200-2300 a.C.), che solo successivamente inizia ad assumere caratteristiche delle cittadelle fortificate cicladiche, come Phylakopi I (2300-2000 a.C.) in cui le case sono costruite da pietre e si inizia ad intravedere una certa organizzazione interna. L'area di assegnazione comprende le isole di Thera, Melos, Paros e Antiparos e sul finire del BAIII anche Naxos e Siphnos.

Le necropoli presentano tombe scavate o ricavate nella roccia in una nuova visione del'architettura funeraria, caratterizzata da una scelta rupestre del tutto nuova, di cui si hanno dei confronti con il sito di Manika, in Eubea, e che forse riflette anche nuovi usi funerari; la ceramica può essere divisa in due grandi gruppi: 1. ceramica incisa caratterizzata da una superficie scura e tra le varie forme si attestano i vasi con becco d'anatra, pissidi coniche e giare; 2 . ceramica dipinta non lucida, la cui decorazione consiste in schemi rettilinei.

L'impianto degli insediamenti e la presenza di fortificazioni attestano l'esistenza di un'avanzata organizzazione sociale, mentre le necropoli forniscono informazioni sulla più articolata stratificazione di tale società, basata su una differenziazione dei ruoli. Sulla base dei rinvenimenti, infatti, è possibile risalire a un'organizzazione basata su classi specializzate di costruttori, scultori, ceramisti, lavoratori di metalli, falegnami, costruttori di navi, navigatori e commercianti che soddisfacevano la necessità della comunità, occupandosi contestualmente della coltivazione della terra, dell'allevamento, della caccia, della pesca, della tessitura e della realizzazione di vasi in vimini per sopperire alle necessità quotidiane.

Queste tre macrofasi costituiscono tre tappe importantissime per la formazione e l'evoluzione della civiltà cicladica, che quindi assume una sua spiccata individualità culturale ${ }^{6}$ tra la fine del Neolitico Recente e l’inizio del Bronzo Antico (3000 a.C. - Antico Cicladico I), e contemporaneamente si assiste alla nascita di una prima forma di proto-urbanizzazione con la cultura di Philakopi, nell’isola di Melos ${ }^{7}$.

\footnotetext{
${ }^{5}$ RENFREW 1972, p.150.

${ }^{6}$ Che assume una valenza ampia del termine, con accezioni anche etniche, economiche, sociali, oltre che culturali in senso stretto.

${ }^{7}$ BARBER 1977, pp.20-23.
} 
In questo periodo i primi insediamenti cambiano carattere; i villaggi neolitici vengono abbandonati a favore di agglomerati che si installano in siti differenti, in prossimità del mare ${ }^{8}$ a maggior controllo di porti naturali e insenature da approdo. Probabilmente il ruolo di 'pacificazione dei mari' attribuita alla civiltà minoica ha determinato una maggiore possibilità di insediamento e sfruttamento delle coste.

All'inizio dell'età del bronzo, i siti appaiono relativamente numerosi, sparpagliati e localizzati in punti naturalmente protetti.

Nel corso dell'Antico Cicladico III (BAIII, 2300-1800 a.C.) si verifica l'apparizione di piccoli insediamenti fortificati. Nelle aree interne si preferiscono zone pianeggianti o in collina. In Tessaglia, Macedonia e Tracia la situazione è differente: i luoghi occupati nel neolitico hanno una continuità anche nell'Antico Cicladico I (Strophilas ad Andros).

Le tecniche di costruzione manifestano una grande stabilità; l'uso dei materiali resta molto simile a quelle del neolitico. Vengono messi in opera sassi e ciottoli per le costruzioni, ampiamente impiegati per le fondamenta, mentre l'alzato alterna mattoni crudi e pietra locale.

La grande maggioranza delle abitazioni è anch'essa costruita in pietra, assieme a materiali naturali per pavimenti e per muri ${ }^{9}$.

Dal punto di vista stratigrafico il passaggio dal Neolitico Recente al Bronzo Antico è visto come una transizione; gli strati, generalmente sono distinti, ma alcuni possono risultare anche sovrapposti o ancor meglio rimossi.

Le case non sono molto differenti da quelle del Neolitico Tardo, rettangolari e sparpagliate; i muri sono costruiti da pietre di piccole e medie dimensioni. Davanti ad ogni casa sono attestati focolari ovali o circolari che presentano una bordatura di terra decorata.

Le comunità erano di scarsa entità, costituite probabilmente da gruppi familiari estesi. La sepoltura dei defunti avveniva in genere in tombe a cista, di solito di piccole dimensioni; le tombe infantili, ma anche quelle degli adulti, sono situate a volte nei pressi di zone abitative, sebbene la maggior parte si localizzi all'esterno in grotte o all'aria aperta, sono normalmente gruppi di cimiteri molto vasti ${ }^{10}$ (necropoli di Calandrini).

Le tombe sono pavimentate da ciottoli con pareti di muretti e lastrone di copertura di forma circolare o ellittica. Nell'Antico Cicladico I le sepolture sono individuali e i corpi sono normalmente in posizione fetale, con le ginocchia contro il mento, ripiegati su di un fianco. Ogni tomba ospitava un solo defunto: evidentemente ogni gruppo di tombe era relativo a un determinato gruppo sociale, probabilmente un clan. Più tardi, nell’ Antico Cicladico II ( 2600 a.C.-2300 a.C. ), le tombe divennero a due o a tre livelli per più sepolture. In questi casi gli scomparti più bassi delle tombe fungevano da ossari. Con l'istituzione di più sepolture, successive l'una all'altra nella stessa tomba, cessò di esistere anche il tipo di organizzazione delle tombe in gruppi, dal momento che ogni tomba era sufficiente ormai a coprire la necessità di ogni piccolo gruppo sociale. Questa evoluzione nell'architettura funeraria pare rispecchiare i mutamenti demografici che ebbero, come risultato, l'aumento delle dimensioni degli insediamenti e, di conseguenza, anche delle necropoli ${ }^{11}$.

I corredi funerari contenevano oggetti che i vivi immaginavano sarebbero serviti ai defunti nell'altra vita: vasi in argilla o di pietre semi-preziose, lame e molte volte grani di ossidiana. Caratteristici tra i corredi funebri erano gli idoli in marmo di varie forme e dimensioni; in essi si traduce il sorgere della forma plastica nel mondo egeo ${ }^{12}$. Vengono rappresentate figure femminili nude, interpretate come dee della fecondità, e allo stesso tempo divinità protettrici dei defunti, dalla valenza ctonia, o persino giocattoli. La produzione di questi idoli si protrae oltre il III millennio: attraverso diversi processi di stilizzazione. I primi

\footnotetext{
${ }^{8}$ Treuil-DARQUE-POURSAT-TOUCHAIS 1989, pp.165-170.

${ }^{9}$ BARBER 1977, p.53.

10 TreuIL-DARQUE-Poursat-TouCHAIS 1989, pp.176-177.

${ }^{11}$ DOUMAS 1994, pp.80-85.

${ }^{12}$ Demargne 2005, p.70.
} 
sono grossolani idoli a forma di violino, con la parte bassa del corpo ridotta a un volume curvo, il collo e la testa ridotti a un cilindro.

Altrettanto caratteristici sono i recipienti in argilla detti a 'padella', che di solito hanno decorazione incisa, a volte lineare con disegni geometrici spirali e a volte con triangoli inscritti ${ }^{13}$.

I vasi dell’Antico Cicladico hanno una superficie levigata e una semplice decorazione incisa rossa o grigio-nera, di solito con un disegno 'a spina di pesce' o inscritta con una serie di triangoli e di spirali. Le forme più abituali sono pissidi cilindriche o scodelle sferiche e coppe, piccoli crateri con piede, oinochoai con collo stretto, vasi piriformi.

Oltre alla decorazione incisa veniva usata anche quella dipinta consistente in semplici disegni geometrici, spesso abbastanza stilizzati. Generalmente i vasi di uso quotidiano hanno sulla superficie esterna del fondo un'impronta di stuoia o di foglia, su cui veniva lasciato asciugare il vaso dopo la sua fabbricazione e prima della cottura.

L'analisi topografica di tale enorme quantità e varietà di siti, dati e rinvenimenti, ha determinato per questo lavoro la scelta di una tipologia insediamentale specifica, da analizzare sia nel contesto topografico, sia in relazione a altre tipologie insedia mentali limitrofe.

\section{Dal database al G.I.S. multi-layers}

Con l'aiuto di un database (Tav. 3) si è cercato di analizzare e di catalogare la maggior parte dei siti cicladici dell'età del bronzo antico per poter stilare una macro-tipologia, il più possibile esauriente, degli insediamenti protostorici del Mar Egeo.

E' stata presa in esame ogni singola isola cicladica e all'interno di ognuna di esse sono state rilevate tutte le emergenze archeologiche note e soprattutto edite, per evitare di utilizzare ricerche archeologiche in atto.

Sono stati identificati 80 siti, di cui 62 sono effettivamente collocati nell'arcipelago delle Cicladi, mentre 18 sono relativi ad insediamenti, sempre databili ad età del bronzo antico, ma che non rientrano direttamente nell'ambito geografico cicladico, seppur molto simili, tanto da permettere confronti su vari livelli.

Le isole prese in considerazione sono quelle di Naxos, Paros, Antiparos, Syros, Delos, Keros, Kea, Kythnos, Melos, Ios, Amorgos; tra gli altri siti stimati Troia, Poliochni nell'isola di Lemnos, Manika in Eubea, alcuni siti di Creta come Ayia Triada, Malia, Fournoi Kofiri, Vasiliki, Tripiti, Koumasa, Nea Roumata, Odigitria, Platanos, Yerokambos, Lerna in Argolide, Emporion nell'isola di Chios.

Il progetto è stato sviluppato per l'impiego di un geodatabase in grado di gestire sia il dato geografico che quello archeologico: i dati in esso raccolti sono stati inseriti nel GIS (Geographical Informational System) in modo che racchiudesse tutte le informazioni relative ai differenti siti archeologici.

Per la realizzazione del geodatabase sono state progettate e realizzate due tabelle: la prima è stata realizzata per contenere l'informazione geografica, ovvero le coordinate geografiche e la definizione geometrica del tipo di dato che nel nostro caso è riassumibile da un 'punto' di localizzazione. La seconda tabella, invece, contiene le informazioni più prettamente archeologiche come per esempio la datazione, la tipologia di sito, la collocazione topografica, i materiali rinvenuti ed altro ancora.

Vista la difficoltà nel reperire una cartografia di base idonea ${ }^{14}$ per questo lavoro, sono state utilizzate delle immagini LANDSAT 7 etm+, realizzate dal satellite francese LANDSAT nel 2000: combinando i differenti spettri, in questo caso si disponeva di sette bande cromatiche (infrarosso visibile, infrarosso semi-visibile, infrarosso invisibile più le 4 bande

\footnotetext{
${ }^{13}$ BARBER 1977, pp.85-90.

${ }^{14}$ Cioè che fosse allo stesso tempo precisa con scala estensibile, con dati topografici ed orografici puntuali e che offrisse una possibilità di visualizzazione sia puntuale che generale.
} 
normali). Attraverso queste immagini è stata impostata una prima base cartografica georeferita, attraverso un sistema UTM $35 \mathrm{~N}$ (trasversale universale di mercatore), e ortocorrette.

Successivamente si è passati all'inserimento di immagini RADAR, questo perché attraverso tali immagini è possibile avere informazioni per quanto riguarda il dato altimetrico seguendo l'ellissoidale relativo allo 0 rapportato al mare. A questo punto è sorto un problema, cioè che tutte queste immagini sono relative allo 0 relativo del mare ma con vette differenti per scala cromatica; per questo si è dovuto riclassificare ad una quota minima (0) e una quota massima (ed è stata scelta la massima tra tutte). Da qui si è ottenuta una carta altimetrica riclassificata per l'intera area, dando vita a una cartografia topografica.

Ultimo passaggio del lavoro, è stato quello di inserire il geodatbase all'interno del GIS in modo da ottenere la sovrapposizione dei diversi siti sulla cartografia realizzata.

Solo a questo punto è stato possibile chiedere al programma, attraverso SQL, cioè Structured Query Language ovvero un linguaggio creato per l'accesso a informazioni memorizzate nei database, una differenziazione tipologica e cronologica dei siti.

Grazie all'analisi degli insediamenti cicladici posso concludere che dei 62 siti cicladici:

- $\quad 16$ appartengono alla prima età del Bronzo Antico (3000 a.C.);

- $\quad 28$ appartengono alla seconda età del Bronzo Antico (2600 a.C.);

- $\quad 3$ appartengono alla terza fase dell'età del Bronzo Antico (2300 a.C.);

- $\quad$ i restanti siti appartengono ai periodi di transizione tra queste tre principali datazioni.

(Tav. 2)

Inoltre, sempre attraverso l'utilizzo del database, ho riscontrato che dei 62 siti cicladici:

- $\quad 43$ sono necropoli;

- 14 sono abitati;

- $\quad 5$ sono fortificazioni.

Delle 43 necropoli:

- 12 appartengono al BAI;

- $\quad 18$ appartengono al BAII;

- $\quad 2$ appartengono al BAIII;

- 6 appartengono al periodo di transizione tra BAI/BAII;

- $\quad 1$ appartiene al periodo di transizione tra BAI/BAIII A;

il resto appartiene sempre ai periodi di transizione.

Dei 14 abitati:

- $\quad 3$ appartengono al BAI;

- $\quad 7$ appartengono al BAII;

- $\quad$ il resto appartiene ai periodi di transizione.

Delle 5 fortificazioni:

- 1 appartiene al BAI;

- 3 appartengono al BAII;

- 1 appartiene al BAIII A.

Le necropoli del Bronzo Antico (3000 a.C.) sono tutte situate su di una collina, o meglio, sui piedi della collina in quanto le sepolture sorgevano all'esterno degli spazi abitativi. Probabilmente le prime inumazioni erano ancora situate nei pressi delle abitazioni, in particolar modo mi riferisco alle sepolture infantili. Successivamente si è verificato uno spostamento graduale delle necropoli.

Il gruppo meridionale delle isole (Paros, Antiparos, Milos, Amorgos, ecc.) si differenzia da quello settentrionale (Kea, Syros, Andros, ecc.) per precipue articolazioni culturali, specialmente per quanto riguarda i tipi di tombe, differenze che vengono ad annullarsi nella compenetrazione osservabile nell'isola di Nasso. 
Le isole del Sud mostrano che il tipo di tomba generalizzato era quello a cista trapezoidale, che è da ritenere il più antico, con tendenza spiccata al seppellimento collettivo; mentre nel gruppo settentrionale erano specialmente in uso le tombe a grotticella artificiale con pozzetto in accesso o camere rettangolari scavate nella roccia con copertura a falsa volta e lastroni (tipiche quelle di Chalandrini a Syros), nelle quali si praticava esclusivamente il seppellimento individuale.

In particolare per le necropoli descritte nel database quelle del Bronzo Antico I sono tutte costituite da tombe a cista con singola sepoltura, mentre quelle del Bronzo Antico II, presentano una doppia sepoltura, nel senso che al di sotto di quella più recente sono attestati $i$ resti di quella più antica. Le necropoli che appartengono al periodo di transizione tra BAI/BAII presentano un corredo molto più ricco delle precedenti ed inoltre alcune di esse mostrano fosse con piano trapezoidale.

Dall'analisi degli abitati si è potuto rilevare che solitamente gli insediamenti erano costituiti da piccoli gruppi d'abitati, in cui i muri erano edificati con pietra locale ed argilla. Tale strutturazione riflette sicuramente l'organizzazione sociale in clan differenziati ma tra loro imparentati.

La maggior parte delle abitazioni del Bronzo Antico sono costituite da due o più ambienti che si presentano rettangolari (Grotta, Naxos e Kastri-Syros); inoltre anche nel Bronzo Antico II le case risultano addossate alle fortificazioni.

Nel corso dell'evoluzione dell'architettura le costruzioni hanno incominciato ad includere degli spazi speciali per le attività domestiche, come l'area adibita alla cucina, all'immagazzinamento della ceramica, come nel caso del sito di Skarkos nell'isola di Ios, che presenta edifici rettangolari costruiti con una tecnica muraria particolarmente precisa ed ordinata. Inoltre si è riscontrato che nell'età del Bronzo Antico II (2600 a.C.) gli ambienti cominciano ad assumere nuove forme, ad esempio nell'isola di Andros il sito di Strophila presenta edifici che hanno una forma absidata, ciò si è verificato anche nell'abitato di Grotta a Naxos.

Relativamente alle opere di fortificazione (Tav. 3), inizialmente le cittadelle protocicladiche non presentavano alcuna fortificazione, indice di sicurezza e dell'assenza di un antagonismo all'affermarsi indipendente della Civiltà Cicladica. Inoltre proprio dall'analisi degli insediamenti si è verificato che tutti i siti dell'età del bronzo sono stanziati su di una collina, ciò vuol dire che le popolazioni cercavano inizialmente dei luoghi naturalmente difensibili.

Molto spesso le prime fortificazioni, che risalgono all'età del Bronzo Antico II (2600 a.c.), si innescano al di sopra della fortificazione naturale; generalmente queste consistono di mura realizzate con pietra locale di medie dimensioni e sono protette da dei bastioni semicircolari.

Di particolare rilevanza sono le fortificazioni dei siti di Kastri nell'isola di Syros, di Panormos nell'isola di Naxos, di Ayia Irini nell'isola di Kea, del Monte Cinto nell'isola di Delos e infine di Strophilas nell'isola di Andros; generalmente questa tipologia di fortificazione ingloba gran parte della collina.

La cultura materiale delle Cicladi rispecchia un po’ la diversità di ideologia funeraria che riflette probabilmente una distinta provenienza dei primitivi occupanti dei due gruppi insulari.

Nelle isole del Nord la ceramica scura lucidata è molto comune, con l'impiego della tecnica decorativa a incisione con motivi geometrici rettilinei (triangoli, spine di pesce) o con spirali variamente congiunte.

Nella forma dei vasi si evince un netto richiamo alla Troade; la maggior parte del materiale rinvenuto negli insediamenti è costituito da pissidi globulari o cilindriche, variamente ornate, in genere, con spirali ricorrenti. Una forma caratteristica delle Cicladi è 
rappresentata da un oggetto fittile discoidale, la cosiddetta 'ceramica a padella', munito da un manico bipartito, la cui destinazione è incerta.

La decorazione dipinta, nei più antichi vasi cicladici (semplici motivi geometrici in bianco su fondo scuro, sostituiti ben presto da motivi in nero su un'ingubbiatura chiara) si riallaccia a quella greco-continentale.

Numerosi sono i vasi in pietra attestati, generalmente realizzati con la tecnica in cui furono esperti i Cicladici e che sta alla base dello sviluppo della loro arte figurativa. L'abrasione e la graduale levigatura del marmo creano in questi vasi superfici sobrie.

La produzione dei vasi in pietra costituiva, insieme a quella degli idoli di marmo, una delle fonti principali per i traffici cicladici intermediterranei, oltre ovviamente al commercio di osidiana e marmo allo stato grezzo e probabilmente dei metalli presenti sulle isole (argento, bronzo e stagno). I tipi di vasi maggiormente riscontrati sono le oinochòai, i pithoi, le anfore dal corpo globulare e il forte becco orizzontale.

Dall'analisi nel G.I.S. è venuto fuori che il sistema territoriale integrato delle isole settentrionali, come quelle di Keros, Syros, Kea ecc., non è gerarchizzato, cioè l'abitato e la fortificazione dovevano avere un diretto rapporto.

Non abbiamo infatti una serie di piccoli abitati che fanno riferimento ad una fortificazione e poi molteplici aree funerarie per ogni abitato, bensì troviamo una grande e ricca necropoli, certamente di riferimento diretto di abitato e fortificazione, evidenziando che esiste già nel Bronzo antico la possibilità di siti funerari articolati e ampi, come troveremo successivamente in tutto il bacino mediterraneo orientale.

Invece per quanto riguarda le isole meridionali ci troviamo di fronte ad un sistema gerarchico organizzato territorialmente in cui più abitazioni ed insediamenti sparsi fanno capo ad numero maggiore di necropoli. Infatti, ad esempio a Naxos, alle necropoli dovevano far capo sia abitanti dei villaggi che coloro che erano stanziati in modo sparso nel territorio e che inoltre ogni abitato doveva far riferimento a più aree funerarie. Dal punto di vista gerarchico invece gli abitati dovevano poi far capo al sito fortificato, che fungeva quindi da controllo e riferimento anche dello spazio geografico circostante, oltre che di aree marittime. Questo tipo di modello gerarchizzato dei siti è stato riscontrato anche per l'isola di Creta.

Dalla lettura delle piante del G.I.S. si evince che l'isola di Naxos è sicuramente la più significativa nel BA (Tav. 4), sia per estensione sia per importanza dei dati ottenuti, fungendo, molto probabilmente, da area centrale per per questo periodo, ed in special modo in riferimento al BAIII. Infatti si è riscontrato che l'isola presenta il maggior numero di siti, la pianta degli abitati mostra con chiarezza come questi dovevano far capo a più aree funerarie, perché relativamente di numero minore rispetto alle necropoli ed inoltre come alle necropoli facessero capo non solo villaggi vicini ma anche quelli stanziati in modo sparso nel territorio.

Le fortificazioni assumono un ruolo particolare, perché nell'età del Bronzo Antico, o meglio, nella prima fase del Bronzo Antico, non esistono fortificazioni, indice sicuramente di maggiore sicurezza, ma anche di una percorrenza più limitata delle rotte marittime. Ma nel momento in cui cominciano ad apparire le prime fortificazioni, probabilmente non solo avevano il compito di difendere il territorio, ma spesso erano realizzate in modo da poter essere viste da chi veniva dal mare, perché a ridosso della costa. 


\section{Bibliografia}

Barber R. N. L., The Cyclades in the Bronze Age, London 1977.

Barber R. N. L., Macgillivray J.A., Early Cycladic period: matter of definition and therminology, in AJA, 84, 1980, pp.141-157.

Broodbank C., An island archeology of the early Cyclades, Cambridge 2000.

Cullen T., Aegean Prehistory, in AJA Supplement I, 2001, pp.19-76.

Davis J., Fortification at Ayia Irini, Keos: evidence for History and relative chronology, B.A. University of Akron, 1972.

Davis J., The island of the Aegean, in Aegean Prehistory, I, 2001, pp. 19-76.

Davis J., Cherry J.F., Papers in Cycladic Prehistory, Los Angeles 1979.

Demargne P., Arte egea, Milano 1964.

Dickinson O., The Aegean Bronze Age, Cambridge 1994.

Doumas C., Notes of early Cycladic architecture, in AA, 1972, pp.151-170.

Doumas C., Early Bronze Age Burial Habits in the Cyclades, Goteborg 1977.

Doumas C., EBA in the Cyclades: continuity or discontinuity?, French-Wardle 1988.

Doumas C., L’Egeo durante la prima età del bronzo: dai Cicladici ai cretesi, in Guilaine J., Settis S. (eds), Storia d’Europa, Preistoria e antichità, Torino 1994, pp.355-371

Karantzali E., Le Bronze Ancien dans les Cylades et en Crete, Oxford 1996.

Harding A.F., European Societies in the Bronze Age, Cambridge 2000.

Macgillivray J.A., Barber R.N.L., The Prehistoric Cyclades, Edinburgh 1984.

Marthari M.E., The early bronze Age in the Cyclades in the light of Recent research at settlement site, Atene 1999.

Renfrew C., The emergence of civilization: the Cyclades and the Aegean in the third millennium BC, London 1972.

Renfrew C., The Cycladic spirit, London 1991.

Sampson A., Early Hellenic contact with the Cyclades during the EBA 2, in R. Laffineur (ed.), Annales d'archólogie égéenne de l'Université de Liìge 2 [Aegaeum 2] (Liìge 1988) 5-10.

Treuil R., Darque P., Poursat J.-C., Touchais G., Les civilisation égéénnes du néolitique et de l’Age du Bronze, Paris 1989. 
Frankfurter elektronische Rundschau zur Altertumskunde 12 (2010)

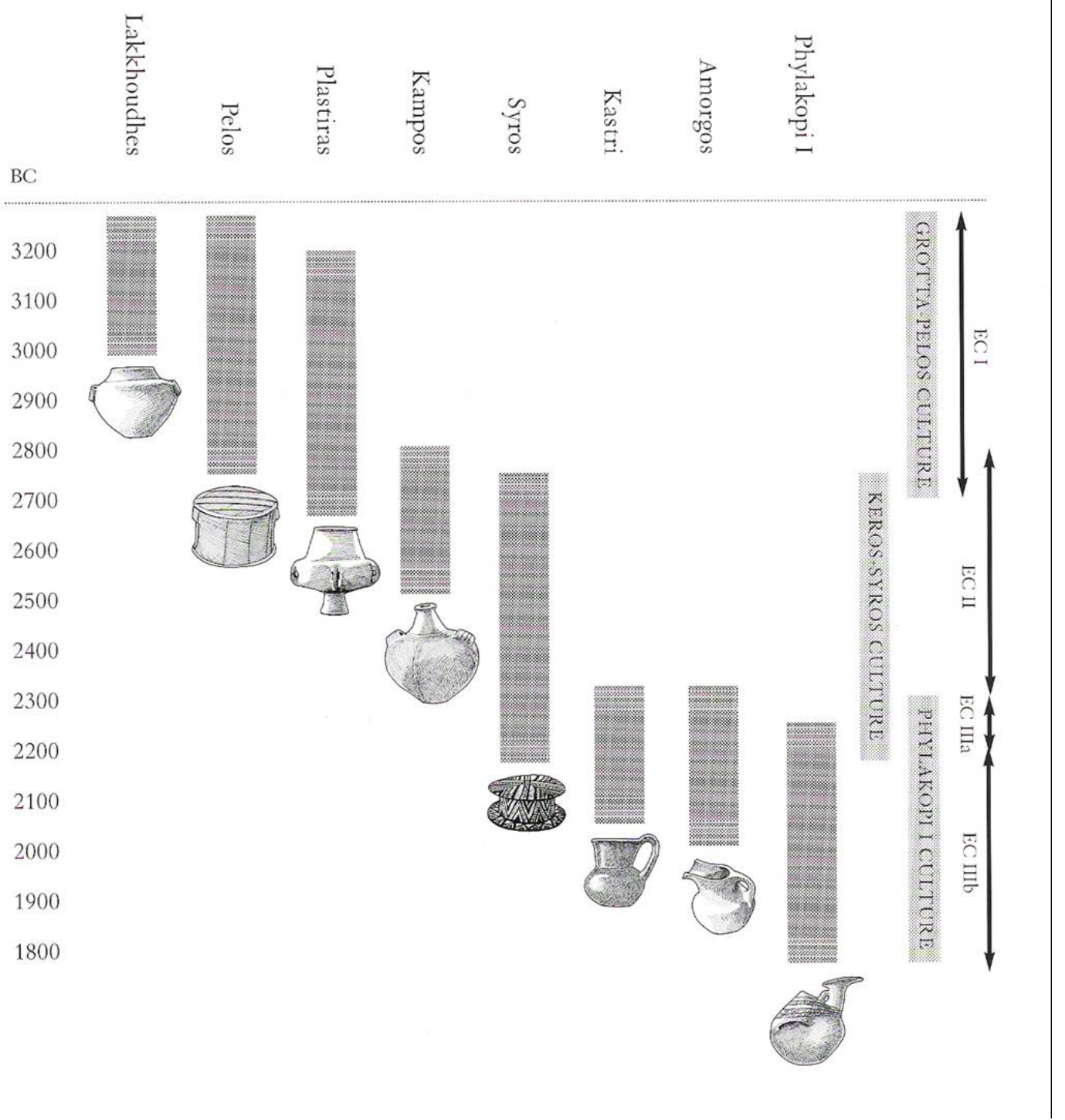

Tav. 1: Tavola cronologica delle culture cicladiche (Barber R.N.L., 1977).

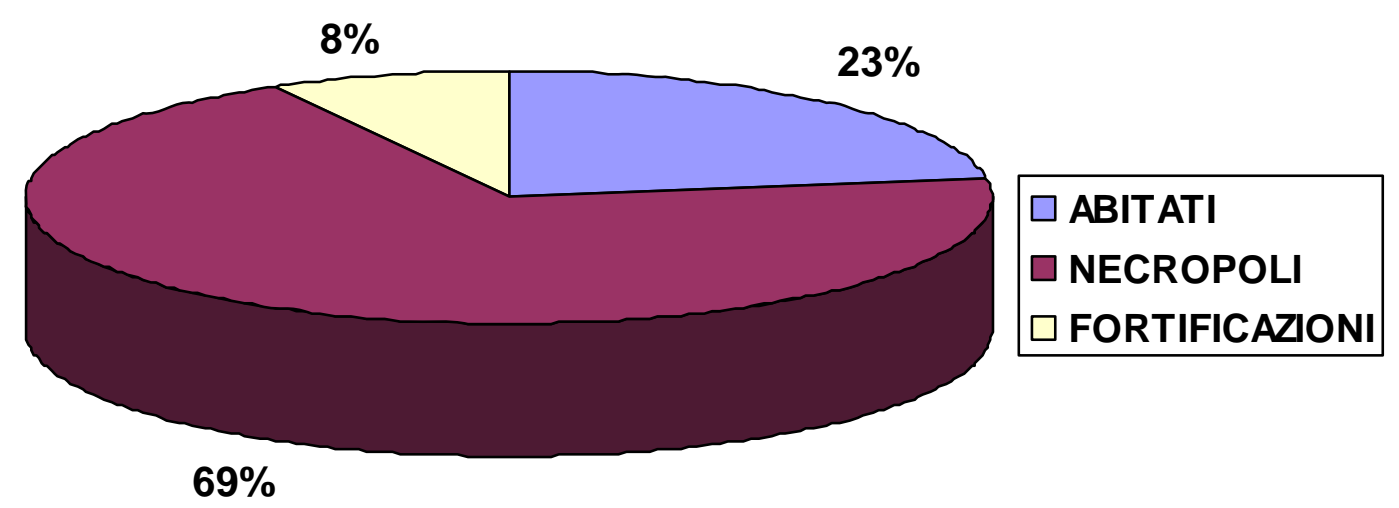

Tav. 2: Grafico dei siti cicladici (by Serena Torello Di Nino) 
Frankfurter elektronische Rundschau zur Altertumskunde 12 (2010)

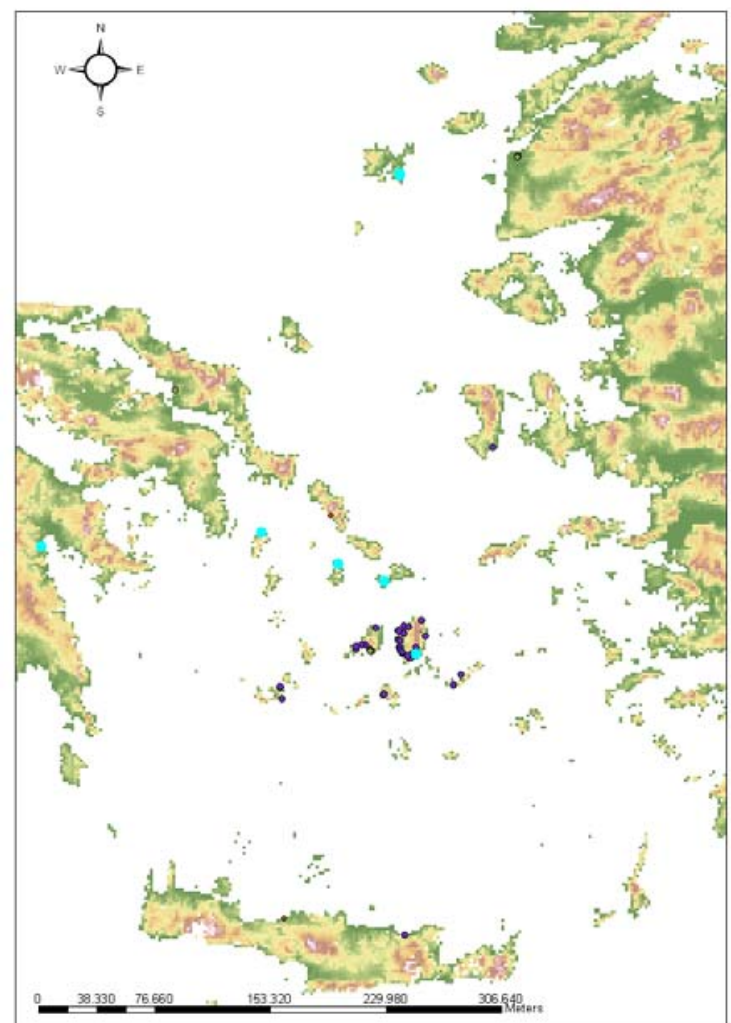

Tav. 3: Pianta delle fortificazioni (by Serena Torello Di Nino).

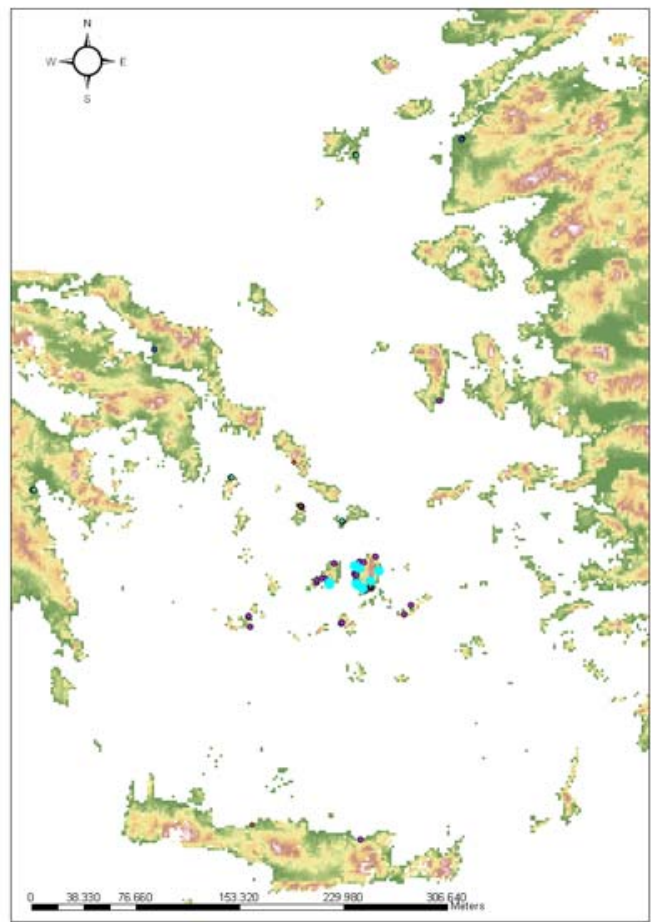

Tav. 4: Pianta cronologica relativa al BA (by Serena Torello Di Nino) 
Frankfurter elektronische Rundschau zur Altertumskunde 12 (2010)

\begin{tabular}{|c|c|c|c|c|c|c|}
\hline \multicolumn{7}{|c|}{ CRONOLOGIE A CONFRONTO } \\
\hline CHRONOLOGY & CYPRIOT & MINOAN & $\begin{array}{c}\text { CYCLADIC } \\
\text { cultures }\end{array}$ & $\begin{array}{l}\text { HELLADIC / } \\
\text { MYCENEAN }\end{array}$ & EGYPT & AGES \\
\hline Before 7000 & $\begin{array}{l}\text { Paleolithic } \\
\end{array}$ & & & & & Paleolithic \\
\hline \multirow[t]{2}{*}{$7000-6000 \mathrm{BC}$} & $\begin{array}{c}\text { Neolithic I (Kirokitia } \\
\text { culture) }\end{array}$ & & & & & Neolitic \\
\hline & cd. Lacuna & & & & & \\
\hline $4500-3800 \mathrm{BC}$ & $\begin{array}{c}\text { Neolithic II } \\
\text { (Sotira culture) }\end{array}$ & Neolithic & Neolithic & Neolithic & & \\
\hline $3900-2500 \mathrm{BC}$ & Chalcolithic & $\begin{array}{c}\text { Sub } \\
\text { Neolithic }\end{array}$ & $\begin{array}{l}\text { Pelos Cult. } \\
(3200-2700)\end{array}$ & Sub Neolithic & $\begin{array}{c}\text { Pre and Proto dynastic } \\
\text { Dyn I-III } \\
3100-2612 \\
\end{array}$ & Chalcolithic \\
\hline $3000-2075 \mathrm{BC}$ & Early Cypriot I & \multirow[t]{3}{*}{$\begin{array}{l}\text { Early } \\
\text { Minoan }\end{array}$} & $\begin{array}{l}\text { Keros-Syros } \\
(2800-2200)\end{array}$ & \multirow[t]{2}{*}{$\begin{array}{c}\text { Early } \\
\text { Helladic }\end{array}$} & $\begin{array}{c}\text { Antico Impero } \\
\text { Dyn.IV-VI } \\
2612-2181 \\
\end{array}$ & \multirow[t]{3}{*}{$\begin{array}{c}\text { Early } \\
\text { Bronze A. }\end{array}$} \\
\hline $2100-2000 \mathrm{BC}$ & Early Cypriot II & & \multirow[t]{3}{*}{$\begin{array}{l}\text { Philakopi } \\
(2300-1800)\end{array}$} & & $\begin{array}{c}\text { I Inter. Per. Dyn. VII-X } \\
2180-2040\end{array}$ & \\
\hline $2000-1900 \mathrm{BC}$ & Early Cypriot III & & & \multirow{4}{*}{$\begin{array}{l}\text { Middle } \\
\text { Helladic }\end{array}$} & \multirow{4}{*}{$\begin{array}{c}\text { Medio Impero } \\
\text { Dyn. XI-XIII } \\
\text { 2133-1625 }\end{array}$} & \\
\hline $1900-1800 \mathrm{BC}$ & Middle Cypriot I & \multirow{4}{*}{$\begin{array}{l}\text { Middle } \\
\text { Minoan }\end{array}$} & & & & \multirow{3}{*}{$\begin{array}{c}\text { Middle } \\
\text { Bronze A. }\end{array}$} \\
\hline $1800-1725 \mathrm{BC}$ & Middle Cypriot II & & & & & \\
\hline 1725-1650 BC & Middle Cypriot III & & & & & \\
\hline $1650-1475 \mathrm{BC}$ & Late Cypriot I & & & \multirow{3}{*}{$\begin{array}{l}\text { Late H-Myc I } \\
\text { Myce II-III } \\
\text { Mycenean III }\end{array}$} & $\begin{array}{l}\text { II Interm. P. } \\
\text { Dy.XIV-XVII }\end{array}$ & \multirow[t]{2}{*}{$\begin{array}{c}\text { Late Bronze } \\
\text { A. }\end{array}$} \\
\hline $1475-1225 \mathrm{BC}$ & Late Cypriot II & \multirow{2}{*}{$\begin{array}{c}\text { Late } \\
\text { Minoan }\end{array}$} & & & \multirow{5}{*}{$\begin{array}{c}\text { Nuovo Impero } \\
\text { From } 11570 \text { - Dyn. } \\
\text { From the XVII in poi }\end{array}$} & \\
\hline $1225-1050 \mathrm{BC}$ & Late Cypriot III & & & & & \\
\hline$\frac{1050-950 \mathrm{BC}}{950-750 \mathrm{BC}}$ & Geometric I/ Proto-G & \multirow{6}{*}{\multicolumn{2}{|c|}{$\begin{array}{c}\text { Sub-Min. } \\
\text { Geometric } \\
\text { Archaic } \\
\text { Classical } \\
\text { Hellenistic } \\
\text { Roman } \\
\text { Late / Medieval }\end{array}$}} & SubMycenean & & Iron Age \\
\hline$\frac{950-750 \mathrm{BC}}{750-480 \mathrm{BC}}$ & & & & & & \\
\hline $480-325 \mathrm{BC}$ & & & & & & \\
\hline $325-50 \mathrm{BC}$ & & & & & & \\
\hline 50 BC-AD 400 & & & & & & \\
\hline after $400 \mathrm{AD}$ & & & & & & \\
\hline
\end{tabular}

Tav. 5: Tabella cronologica (by Serena Torello Di Nino). 